\title{
Study of oscillations with accelerator and reactor neutrinos
}

\author{
Yury Kudenko ${ }^{1,2,3, *}$ \\ ${ }^{1}$ Institute for Nuclear Research of the Russian Academy of Sciences, 117312 Moscow, Russia \\ ${ }^{2}$ Moscow Institute of Physics and Technology, 141701 Moscow Region, Russia \\ ${ }^{3}$ Moscow Engineering Physics Institute (MEPhI), 115409 Moscow, Russia
}

\begin{abstract}
The present status of accelerator and reactor neutrino experiments and new results are presented. A short overview of future projects is also given. The experimental status of sterile neutrino searches is briefly discussed.
\end{abstract}

\section{Introduction}

The discovery of neutrino oscillations $[1,2]$ has provided convincing evidence for non-zero neutrino masses and leptonic mixing. This phenomenon is the first clear example of the new physics beyond the Standard Model (SM). Oscillation data obtained by now in atmospheric, solar, accelerator and reactor experiments are well described in the framework of three active massive neutrinos which flavour eigenstates $v_{e}, v_{\mu}, v_{\tau}$ and mass eigenstates $v_{1}, v_{2}, v_{3}$ with masses $m_{1}, m_{2}, m_{3}$ are related by the Pontecorvo-Maki-Nakagawa-Sakata (PMNS) $3 \times 3$ unitary mixing matrix. This matrix is parametrized by three mixing angles $\theta_{12} \sim 34^{\circ}, \theta_{23} \sim$ $45^{\circ}, \theta_{13} \sim 9^{\circ}$ and a CP-violating phase $\delta_{\mathrm{CP}}$. Non-zero masses appear in oscillations as two independent squared-mass differences between mass eigenstates $\Delta m_{21}^{2}=m_{2}^{2}-m_{1}^{2} \simeq 7.5 \times 10^{-5}$ $\mathrm{eV}^{2}$ and $\left|\Delta m_{32}^{2}\right|=\left|m_{3}^{2}-m_{2}^{2}\right| \simeq 2.4 \times 10^{-3} \mathrm{eV}^{2}$.

The number of light neutrinos constrained by LEP experiments is $N_{v}=2.9840 \pm 0.0082$. However, this result does not exclude existence of sterile neutrinos which could mix with active neutrinos and therefore could modify the $3 \times 3$ mixing matrix. There are three short baseline neutrino oscillation anomalies which do not correspond to the neutrino oscillation paradigm with 3 active neutrinos: 1) a $\bar{v}_{e}$ excess in a $\bar{v}_{\mu}$ beam observed by the LSND experiment [3]; 2) a deficit of $v_{e}$ found in measurements with a radioactive neutrino source by the SAGE [4] and GALLEX [5] experiments, and 3) a deficit of reactor antineutrinos relative to the calculated flux observed in several short baseline reactor experiments [6]. These results, if correct, could be explained by introducing at least one additional squared-mass difference $\Delta m^{2} \geq 1 \mathrm{eV}^{2}$. This requires the existence of at least one extra massive neutrino $v_{4}$ which corresponds to a sterile neutrino in flavour basis.

This brief review covers the recent progress in study of neutrino oscillations. The new results on sterile neutrinos obtained in various experiments are discussed. Next decade projects aiming at the discovery of $\mathrm{CP}$ violation and measurement of $\delta_{\mathrm{CP}}$ and determination of the neutrino mass hierarchy are also outlined.

*e-mail: kudenko@inr.ru 


\section{Current experiments}

\subsection{Results from T2K}

The T2K (Tokai-to-Kamioka) experiment [7] uses an off-axis neutrino beam peaked at 0.6 $\mathrm{GeV}$ directed from J-PARC towards the Super-Kamiokande detector $295 \mathrm{~km}$ away. T2K collects data $\left(v_{\mu}\right.$ and $v_{e}$ events with the neutrino beam and $\bar{v}_{\mu}$ and $\bar{v}_{e}$ events with the antineutrino beam) since 2010 and released updated results reported at several conferences this Summer (see, for example, [9]). The results are based on $14.9 \times 10^{20}$ protons on target (POT) for neutrino mode and $11.2 \times 10^{20}$ POT for antineutrino mode. In 2016, T2K reported [8] that the CP conservation hypothesis $\left(\delta_{\mathrm{CP}}=0\right.$ or $\pi$ ) is excluded at $90 \%$ confidence level (CL). Since then, the number of detected neutrino events was greatly increased and the electron neutrino and antineutrino event selection efficiencies have increased by $30 \%$ and $20 \%$, respectively. The new result on measurement of $\delta_{\mathrm{CP}}$ is shown in Fig. 1 . The best fit value is $\delta_{\mathrm{CP}} \simeq-1.60 \mathrm{rad}$
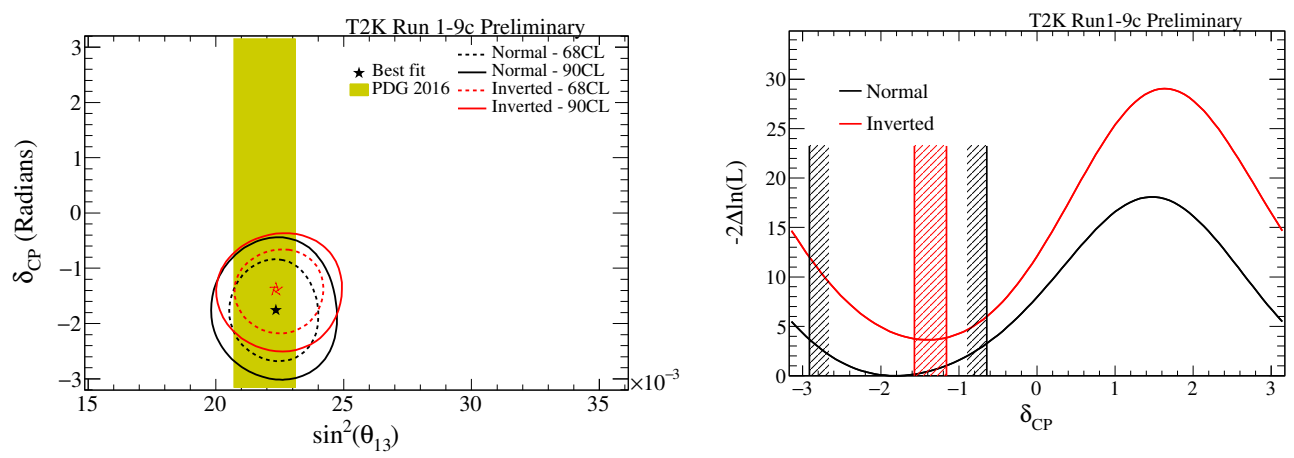

Figure 1. Left: best fit points (stars) and allowed $68 \%$ and $90 \% \mathrm{CL}$ regions of $\sin ^{2} \theta_{13}$ and $\delta_{C P}$ for the normal (black) and inverted (red) mass hierarchy obtained in the $\mathrm{T} 2 \mathrm{~K}$ experiment. Reactor constraint $\pm 1 \sigma$ on $\theta_{13}$ indicated as PDG2016 is used. Right: black and red curves show $2 \Delta \ln (\mathrm{L})$ significance at which each value of $\delta_{\mathrm{CP}}$ is disfavored for normal and inverted mass hierarchy, respectively. Vertical lines limit the allowed regions for $\delta_{\mathrm{CP}}$ values by the $2 \sigma$ confidence level. The best fit point is $\delta_{\mathrm{CP}}=-1.60$ rad for the normal mass hierarchy. The reactor constraint on $\theta_{13}$ is used.

for the normal mass hierarchy, and CP conserving values $\delta_{\mathrm{CP}}=0$ and $\delta_{\mathrm{CP}}=\pi$ lie outside the $2 \sigma$ region. The $\mathrm{T} 2 \mathrm{~K}$ data show a slight preference for the normal neutrino mass hierarchy.

\subsection{Results from $\mathrm{NO} v \mathrm{~A}$}

The NO $v$ A experiment [10] uses the Fermilab NUMI neutrino beam to measure the neutrino oscillations at the distance of $810 \mathrm{~km}$ from the pion production target. The far detector sits $14.6 \mathrm{mrad}$ away from the central axis of the NuMI beam. This off-axis location results in a neutrino flux with a narrow-band energy spectrum centered around $1.9 \mathrm{GeV}$ in the far detector. Such a spectrum emphasizes $v_{\mu} \rightarrow v_{e}$ oscillations at this baseline and reduces backgrounds from higher energy neutral current events. The identical near detector is positioned to maximize the similarity between the neutrino energy spectrum at its location and that expected at the far detector in the absence of oscillations. The experiment accumulated $8.85 \times 10^{20}$ POT in a neutrino mode and $6.9 \times 10^{20}$ POT in antineutrino mode. After selection, $113 v_{\mu}$ candidates were observed in the far detector in the neutrino mode. In the absence of oscillations, 730 events were expected. $65 \bar{v}_{\mu}$ events were detected in the antineutrino mode, 
while 266 events are expected without oscillations. NOvA accumulated $58 v_{e}$ events, including 15 expected background events in the far detector in a muon neutrino beam. In the antineutrino beam, a total of $18 \bar{v}_{e}$ events were detected, corresponding to a significance of $>4 \sigma$ when compared to 5.3 expected background events. Joint analysis of appearance and disappearance data are shown in Fig. 2. The best fit values $\Delta m_{32}^{2}=(2.51+0.12-0.08) \times 10^{-3} \mathrm{eV}^{2}$,

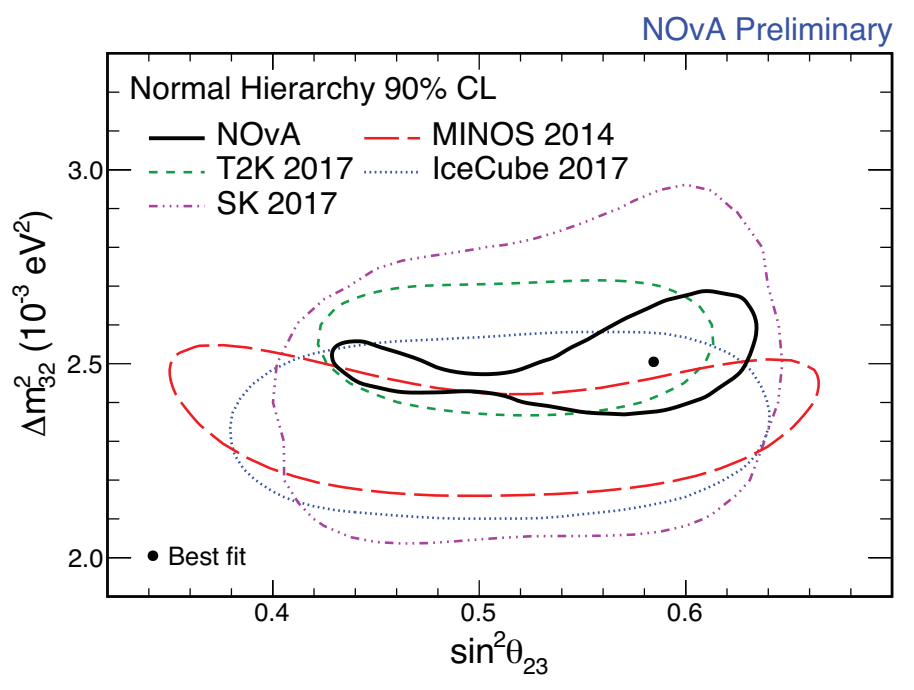

Figure 2. $90 \%$ confidence regions in the plane $\left|\Delta m_{32}^{2}\right|-\sin ^{2} \theta_{23}$ obtained in oscillation experiments NO $v$ A, T2K, Super-Kamiokande (SK), MINOS, and IceCube. Black dot shows the best fit of the $\mathrm{NO} v \mathrm{~A}$ data.

$\sin ^{2} \theta_{23}=0.58 \pm 0.03$ were obtained for the normal mass hierarchy and the upper octant. $\mathrm{NO} v \mathrm{~A}$ result prefers the normal mass hierarchy at $1.6 \sigma$ [11].

It should be also noted that the OPERA experiment recently released its final result [12]. $10 v_{\tau}$ events were observed for $18 \times 10^{19}$ POT, while the expected background is $2.0 \pm 0.4$ events. The $v_{\tau}$ appearance was confirmed with an improved significance level of $6.1 \sigma$. In this experiment, the measurement of $\left|\Delta m_{32}^{2}\right|$ was first time done in the appearance mode. The obtained value $\left|\Delta m_{32}^{2}\right|=\left(2.7_{-0.6}^{+0.7}\right) \times 10^{-3} \mathrm{eV}^{2}$ is in a good agreement with disappearance results from different experiments.

\section{Future long baseline reactor and accelerator experiments}

A rich experimental program is under preparation to answer the fundamental questions in neutrino physics. Is there leptonic $\mathrm{CP}$ violating or not? What is the neutrino mass hierarchy? What is the value of $\theta_{23}$ ? Some answers, or hints, can be obtained from running experiments $\mathrm{T} 2 \mathrm{~K}, \mathrm{NO} v \mathrm{~A}$, IceCube, Super-Kamiokande, and reactor experiments. However, only the next generation of long baseline accelerator experiments, DUNE, T2HK and the reactor experiment JUNO have real chances to discover CP violation in neutrino oscillations and determine the neutrino mass hierarchy. 


\subsection{JUNO}

The long baseline reactor experiment JUNO (Jiangmen Underground Neutrino Observatory) [13] has two main goals: the determination of the neutrino mass hierarchy and the precise measurement of oscillation parameters $\Delta m_{21}^{2}, \Delta m_{31}^{2}$, and $\sin ^{2} \theta_{12}$. The JUNO detector, a $20 \mathrm{kt}$ spherical unsegmented liquid scintillator detector, will be located at a distance of 53 $\mathrm{km}$ from two sites of Yangon and Tanisha, where 6 and 4 nuclear reactors are to be built, respectively. According to the construction plan, the total power of $35.8 \mathrm{GW}$ will be available. The detector will be instrumented with more than 17000 20-inch and about 250003 -inch photo multiplies (PMTs) ensuring a 77\% photocathode coverage. To discriminate between the neutrino hierarchies at $\mathrm{a} \geq 3 \sigma$ level, the energy resolution of this detector is required to be $3 \% / \sqrt{E(\mathrm{MeV})}$ and the absolute energy scale should be calibrated with a precision of $1 \%$. It is expected that after 6 years of data taking JUNO can distinguish between the true and wrong hierarchy hypothesis at a significance level of $4 \sigma$, assuming no systematics. If systematic uncertainties are taking into account (non equal baselines from reactors to the detector, backgrounds, the shape of the reactor antineutrino spectrum), the significance to determine the mass hierarchy is reduced to about $3 \sigma$. The funding of JUNO was approved in 2013, the civil construction begun in 2015, the tunnel are already completed and the detector installation is foreseen to start in 2019. JUNO plans to begin data taking in 2020.

\subsection{DUNE}

The main scientific goals of the Deep Underground Neutrino Experiment (DUNE) [14] are the sensitive test of $\mathrm{CP}$ violation in the leptonic sector, determination the neutrino mass hierarchy, and precise measurements of neutrino oscillation parameters. The proposed liquid argon far neutrino detector will be built deep underground, at a depth of about $1500 \mathrm{~m}$, in the Sanford Underground Research Facility (South Dakota, USA), about 1300 km from Fermilab where a high intensity wide band on-axis muon neutrino (antineutrino) beam with energies of 1-6 GeV will be formed. This neutrino beam will cover the first and the second oscillation maxima which correspond to the neutrino energy of $2.5 \mathrm{GeV}$ and $0.8 \mathrm{GeV}$, respectively. The far detector will consist of four cryostats instrumented with Liquid Argon Time Projection Chambers (LAr TPCs) with a fiducial mass of $40 \mathrm{kt}$. The first $10 \mathrm{kt}$ DUNE liquid argon module adopts a single-phase technology pioneered by the ICARUS T600 detector. The second DUNE far detector module can be constructed on the basis of a dual-phase TPC if its performance is confirmed as a result of activities at the CERN Neutrino Platform. The LAr TPC technology offers excellent capabilities for position and energy resolution and for highprecision reconstruction of complex interaction topologies over a broad neutrino energy range and will provide a powerful complementarity to the large, underground water Cherenkov or scintillator-based detectors. A staged far detector installation approach is adopted. The far detector cavern groundbreaking occurred in 2017 with the installation of the first $10 \mathrm{kt}$ module planned in 2021, second one in 2023. Non-beam physics measurements with 2 modules will start in 2024. First beam at 1.2 MW is expected in 2026. The expected sensitivity to CP violation of DUNE is shown in Fig. 3. To validate that the design and performance of 10 kt TPC's are sufficient to reach the physics goals, two large scale prototypes (a single phase and a dual phase) are currently under construction at CERN. Charged pions and protons will be used to better understand interaction processes in Ar and to optimize the reconstruction technique. Commissioning of prototypes and data taking are expected in the fall of 2018, before the SPS long shutdown. 


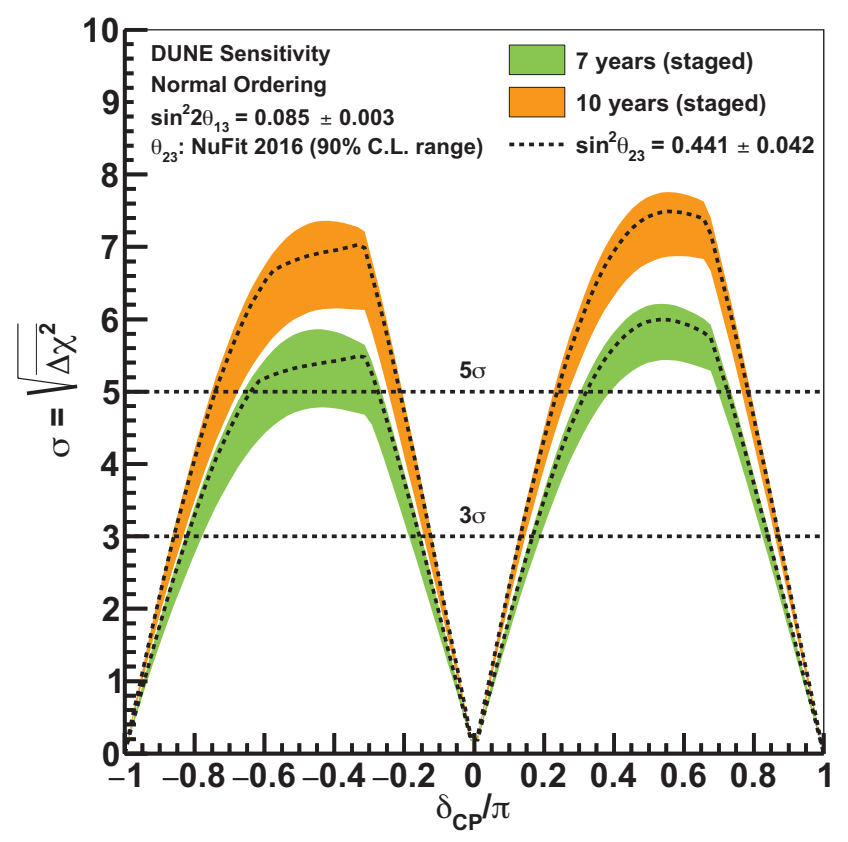

Figure 3. Significance with which the CP violation can be determined in DUNE with exposures of seven (green) and ten (orange) years [15]. The normal mass hierarchy is assumed. The width of the bands corresponds to the $90 \%$ CL range of $\theta_{23}$ values.

\subsection{T2HK and Hyper-Kamiokande}

The main goal of the T2HK (Tokai-to-Hyper-Kamiokande) experiment is the sensitive search for CP violation in neutrino oscillations [16]. A gigantic water Cherenkov HyperKamiokande detector equipped with newly developed high efficiency and high-resolution PMTs will serve as a far detector in this experiment which will use neutrino and antineutrino beams produced at J-PARC upgraded to the power of $\sim 1.3 \mathrm{MW}$. The baseline design includes one $260 \mathrm{kt}$ (186 kt fiducial mass) Cherenkov detector at a distance of $295 \mathrm{~km}$ from J-PARC. The inner detector region of the tank is viewed by 40,000 PMTs that provides a $40 \%$ photo-cathode coverage. As in $\mathrm{T} 2 \mathrm{~K}$, the $2.5^{\circ}$ off-axis beam tuned to the first oscillation maximum will be used. For the estimation of the sensitivity, an integrated beam power of $13 \mathrm{MW} \times 10^{7} \mathrm{sec}$ is assumed, corresponding to $2.7 \times 10^{22}$ protons on target with a $30 \mathrm{GeV}$ J-PARC beam, or to about 10 years of data taking with one tank. For the $v: \bar{v}$ ratio of 1:3 the expected number of events will be approximately the same for both modes: $\sim 2000 v_{\mu} \rightarrow v_{e}$ events with the signal-to-background ratio of about 4:1 and $\sim 1900 \bar{v}_{\mu} \rightarrow \bar{v}_{e}$ events with the signal-to-background ratio of $>1.5$ [17]. Fig. 4 shows the T2HK potential of exclusion of 


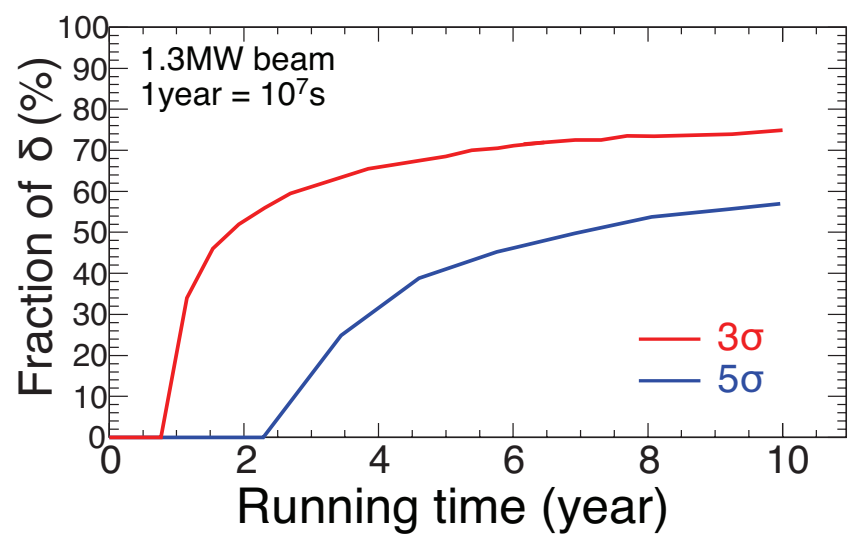

Figure 4. Fraction of $\delta_{C P}$ for which $\sin \delta_{C P}=0$ can be excluded with more than $3 \sigma$ (red curve) and $5 \sigma$ (blue curve) significance for normal mass hierarchy as a function of the running time. The ratio of the neutrino and anti-neutrino running time is 1:3.

$\sin \delta_{C P}=0$. The construction of the Hyper-Kamiokande is expected to begin in 2019 and the detector to be operational by 2026 .

\section{Sterile neutrinos}

A few new results on searches for sterile neutrinos were released within one year. The status of this problem is briefly outlined below.

Test of the LSND/MiniBooNe anomaly. The analysis of all available data presenting the tests of this anomaly is given in [18]. The most interesting is the tension between the appearance and disappearance data shown in Fig. 5. Bounds on $\left|U_{e 4}\right|$ and $\left|U_{\mu 4}\right|$ obtained from the electron antineutrino and muon neutrino disappearance data lead to a quadratic suppression of the effective amplitude $\sin ^{2} 2 \theta_{\mu e}$ responsible for the $v_{\mu} \rightarrow v_{e}$ appearance. Constraints from the disappearance data challenge the explanation of the LSND/MiniBooNe anomaly in the appearance channel in terms of sterile neutrino oscillations. This figure shows that there is no overlapping between the parameter region favored by LSND and MiniBooNe and the strong exclusion limit from disappearance data, mainly due to the recent data from MINOS/MINOS ${ }^{+}$and IceCube. MiniBooNe accumulated more data and recently updated the analysis revealing that the excess of electron-like events, observed in both the neutrino and antineutrino modes, is now a $4.8 \sigma$ effect [19]. The best combined with the LSND data neutrino oscillation fit occurs at $\left(\Delta m^{2}, \sin ^{2} 2 \theta\right)=\left(0.041 \mathrm{eV}^{2}, 0.958\right)$. However, this best fit point was already excluded by the OPERA and ICARUS experiments. It seems that the crucial test of the LSND/MiniBooNe anomaly will be performed by the Fermilab short-baseline neutrino (SBN) program [20].

Test of the reactor anomaly. New constraints on $\bar{v}_{e}$ disappearance into sterile neutrinos were recently obtained in reactor experiments NEOS [21], Neutrino-4 [22], DANSS [23], PROSPECT [24], and STEREO [25]. The results obtained in these experiments are independent from neutrino flux predictions and insensitive to the predicted spectrum shape. The ex- 


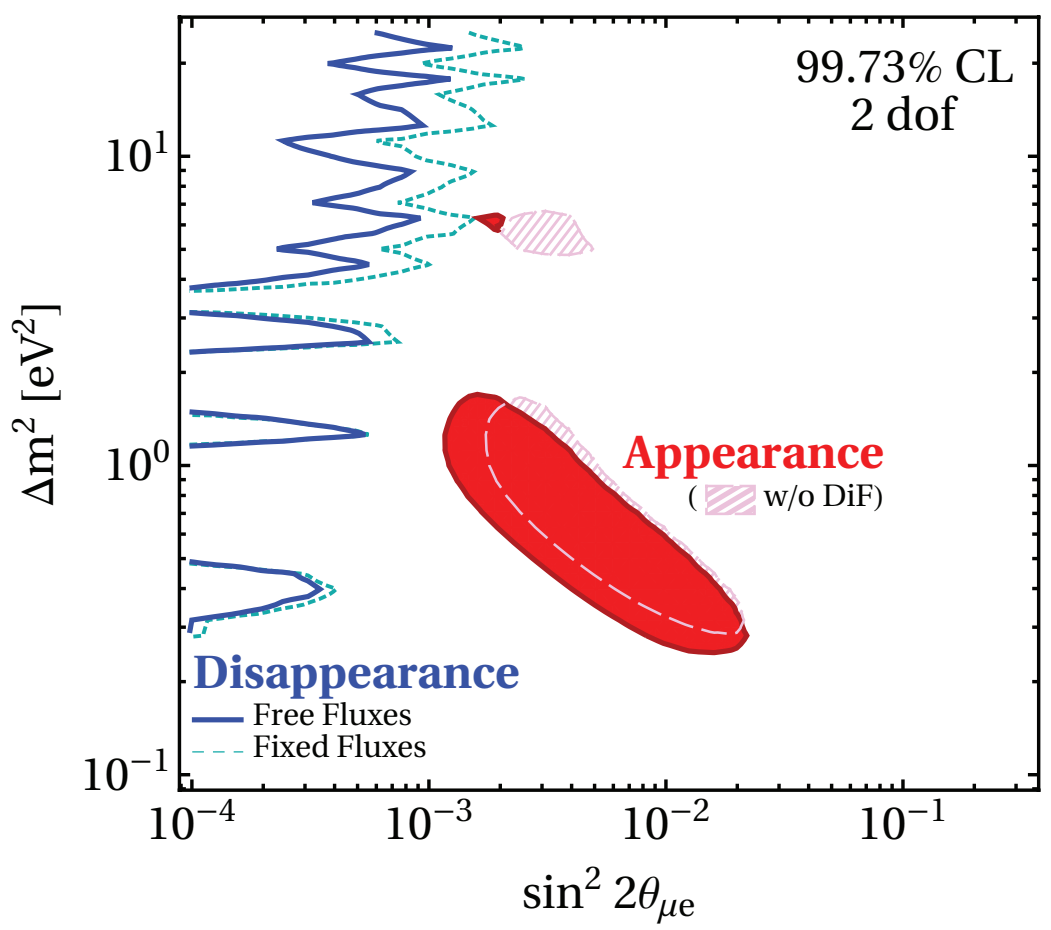

Figure 5. Appearance versus disappearance data in the plane $\Delta m^{2} 41-\sin ^{2} 2 \theta_{\mu e}=4\left|U_{e 4} U_{\mu 4}\right|^{2}$ [18]. The blue curves show limits $(99.73 \% \mathrm{CL})$ from the disappearance data using free reactor flux (solid) and fixed reactor flux (dashed). The shaded contours are based on the LSND appearance data.

clusion contour obtained in the DANSS experiment is shown in Fig. 6. All mentioned above experiments exclude the original reactor anomaly best fit $\Delta m_{41}^{2}=2.4 \mathrm{eV}^{2}$ and $\sin ^{2} 2 \theta=0.14$.

\section{Conclusion}

The current oscillation data is successfully described by the standard 3-flavor neutrino scheme, but large deviations from this paradigm are allowed. We still do not know how the neutrino masses are ordered. Do we have the so-called normal mass hierarchy or the inverted hierarchy? Is there CP violation in the lepton sector? The answers to these questions are expected to be obtained in current and future accelerator and reactor oscillation experiments. Intriguing anomalies that cannot be accommodated within the three-neutrino scheme and point to existence of sterile neutrinos are being addressed by many experiments using reactor, accelerator, and atmospheric neutrinos. There are good prospects that these dedicated experiments will start to resolve the problem of sterile neutrinos in a very near future.

\subsection{Acknowledgments}

This work was supported in part by the RFBR/JSPS grant \# 17-52-50038. 


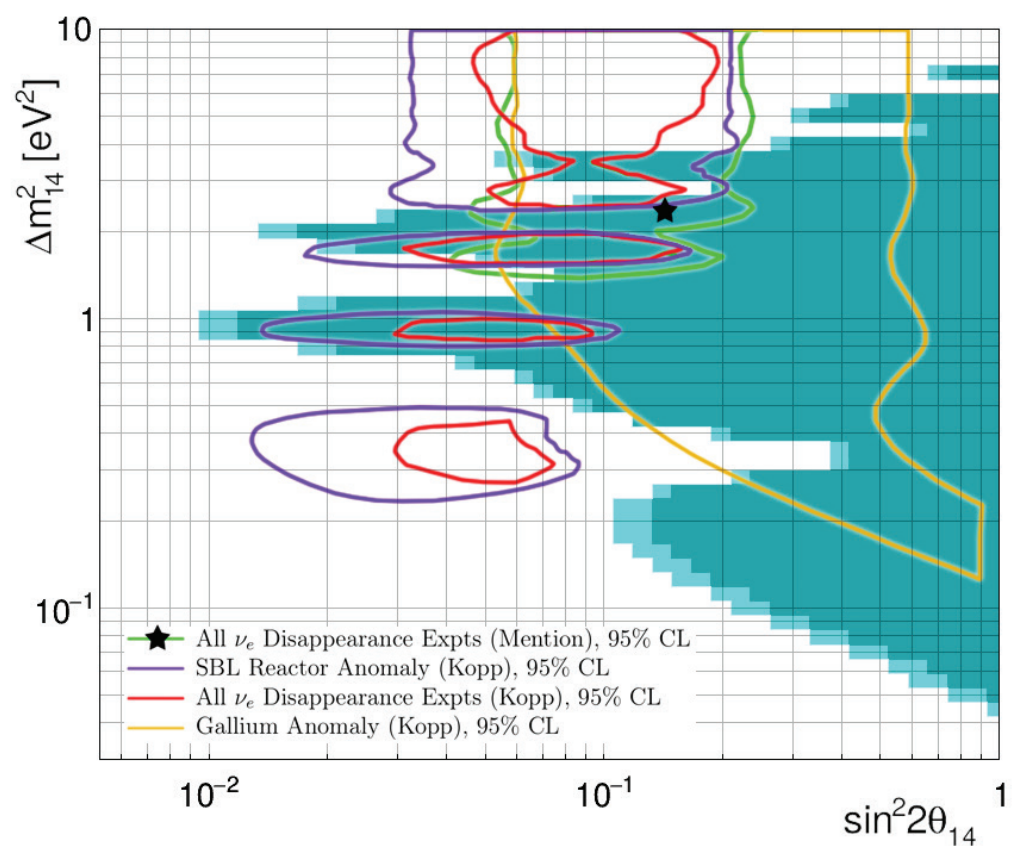

Figure 6. Exclusion contour obtained in the DANSS experiment [23]. The shaded areas show the $90 \%$ CL (cyan) and 95\% CL (dark cyan) excluded regions. Curves show the allowed regions from reactor disappearance experiments. The star shows the best fit point from the reactor and gallium anomalies.

\section{References}

[1] T.Kajita, Rev. Mod. Phys. 88030501 (2016).

[2] A.B.McDonald, Rev. Mod. Phys. 88030502 (2016).

[3] A.Aguilar et al., Phys. Rev. D64 112007 (2001).

[4] J.Abdurashitov et al., Phys. Rev. C73 045805 (2006).

[5] W.Hampel et al., Phys. Lett. B420 114 (1998).

[6] G.Mention et al., Phys. Rev. D83 073006 (2011).

[7] K.Abe et al., Nucl. Instrum. Meth. A659 106 (2011).

[8] K.Abe et al., Phys. Rev. Lett. 118151801 (2017).

[9] M.Wascko, talk at NEUTRINO2018, 4-9 June 2018, Heidelberg, Germany, https://zenodo.org/badge/DOI/10.5281/zenodo.1286752.svg.

[10] NOvA Technical Design Report No. FERMILAB-DESIGN-2007-1.

[11] J.Bian, talk at ICHEP2018, 4-11 July 2018, Seoul, Korea.

[12] N.Agafonova et al., Phys. Rev. Lett. 120211801 (2018).

[13] T.Adam et al., arXiv: 1508.07166 [hep-ex] (2015).

[14] R.Acciarri et al., arXiv:1512.06148 [physics.ins-det] (2015).

[15] D.Brailsford, arXiv:1804.04979 [physics.ins-det] (2018).

[16] K.Abe et al., arXiv:1412.4673 [physics.ins-det] (2014).

[17] K.Abe et al., arXiv:1805.04163 [physics.ins-det] (2018).

[18] M.Dentler et al., arXiv:1803.10661 [hep-ph] (2018). 
[19] A.A.Aguilar-Arevalo et al., arXiv:1805.12028 [hep-ex] (2018).

[20] M. Antonello et al., arXiv:1503.01520 (2015).

[21] Y.J.Ko et al., Phys. Rev. Lett. 118121802 (2017).

[22] A.Serebrov et al., arXiv:1708.00421 [physics.ins-det] (2017).

[23] I.Alexeev et al., arXiv:1804.04046 [hep-ex] (2018).

[24] J.Ashenfelter et al., arXiv:1806.02784 [hep-ex] (2018).

[25] H.Almazan et al., arXiv:1806.02096 [hep-ex] (2018). 